\title{
STUDY OF THE SHORT-TERM GENERATION BLOCK FREQUENCY FORECASTING OF A POWER GENERATION UTILITY USING ARTIFICIAL NEURAL NETWORK
}

\author{
Shailendra Singh ${ }^{1}$, R P Payasi ${ }^{2}$, S K Sinha ${ }^{3}$ \\ ${ }^{1} M$. Tech. Scholar, Department of the Electrical Engg, KNIT, Sultanpur, UP, India \\ ${ }^{2}$ Professor, Department of the Electrical Engg, KNIT, Sultanpur, UP, India \\ ${ }^{3}$ Professor, Department of the Electrical Engg, KNIT, Sultanpur, UP, India
}

\begin{abstract}
Frequency forecasting a very vital aspect in power system operation and control. For power generation utilities the computation of the frequency forecasting is crucial for administering power generation and trading optimally. Beside this, frequency of the grid is the most vital and decisive parameter for the appraisal of the entire power system network safety, and to estimate the grid power quality. Frequency of the power system corresponds to power generation balance to any point of time with respect to load/demand. The power generation inequality/imbalance leads to differ frequency from its nominal value i.e. $50 \mathrm{~Hz}$ in our country. Larger deviation in frequency can sabotage power system operation severely in the form of Grid turmoil along with the tripping of the Generators and turbines connected to Grid which can eventually leads to grid collapse. Forecasting the frequency ahead in time is desired to avert all these problems instigating on the frequency deviations. Importance of the frequency perceived with advent of the ABT, by imposing the tariff to power generating utilities sensitive to the frequency. Part of this tariff is paid for UI (Unscheduled Interchange) of power supplied in deviation with scheduled power.
\end{abstract}

In traditional approaches, frequency prediction algorithms prepared based on pattern matching of the previous frequencies, but here in this paper an algorithms is prepared which will include the various other non linear parameters also such as actual power generated, load demand in term of scheduled power generation allocated to particular utility. This deficit/surplus power is taken as one of the key factor for forecasting the frequency to a fair degree of accuracy. ANN's ability to provide most accurate forecasting results with smaller processing time is utilized here in this paper.

In this work, we will study short-term frequency prediction for a thermal power generating station station with the help of ANN. We will utilize the past two year data of plant's suggested generation, declared capacity and frequency for each block of 15 minutes to train the Artificial Neural Network.

Keywords: ABT, Unscheduled Interchange, NRLDC Artificial Neural Network, Load Dispatch

\section{BACKGROUND}

Frequency forecasting in power system is quite tedious job as nature of frequency is stochastic. It varies with declared capacity of the station, grid network frequency demand, surplus/deficit power in system. Besides this, it also depends on frequency value in previous blocks. Considering these all-crucial parameter numerous forecasting techniques evolved [2], [3] considering stochasticity of the frequency.

In conventional methods, such as time series, regression theory model, Kalman Filter and Spectral Expansion technique considered frequency values of the previous times with known per day or per session basis periodicities. Authentic frequency forecasting is not viable with these methods, as they do not adequately reassemble non-linearity between the frequency and various other parameters associated with it.

Apart from this, these approaches demonstrate larger processing time, sluggish performance and incorrect forecasting due to rapidly season change. Artificial Intelligence (AI) based approaches such as ANN, Genetic Algorithams, Experts Systems and fuzzy logic have shown their excellence over the conventional methods of forecasting. The Artificial Neural Network learns from experience and easily embodied to adopt the non-linear relationship between frequency and other factors associated with historical data.

ANN takes smaller computational time in comparison with conventional approaches and provides more accurate prediction results in comparison to traditional approaches. Different forecasting approaches with ANN presented in literature [1], [2] by considering the frequency demand forecasting.

Hence, with application of the ABT for enforcing charges on power drawl that is sensitive to the system frequency, power utility operator focused on designing predicting generation block frequency for earning UI charges and scheduling of the generators accordingly. 


\section{SALIENT FACTOR FOR FORCASTING}

A classical method of the frequency prediction block diagram is shown in Fig.1.Based on the span and desired accuracy of the forecasted frequency, the several other features such as effect of environment /external factor may also be integrated for optimal utilization of available data for ANN training. Historical data of the frequency value of previous time must provide along with the power generation demand/schedule, actual generation and capacity of the generation utility.

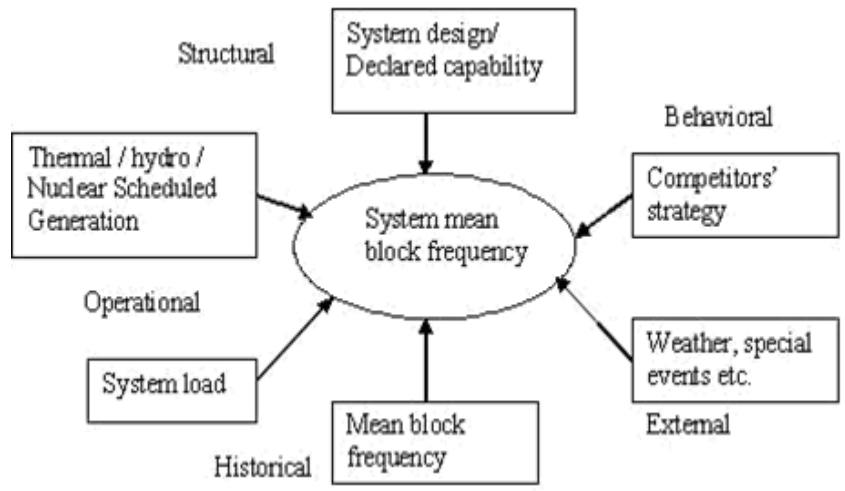

Fig.1: Factor affecting frequency prediction.

\section{PROBLEM FORMULATION}

Schedule of power generation to the any power generation utility is given by Regional load dispatch center in blocks of 15 minute. This schedule update on RLDC site in span of every 4-5 block of generation based on actual power demand in grid. Individual unit of any power station generate power keeping in mind the unit schedule of that block along with frequency for earning additional UI charges by generating more than schedule power in case of under frequency and less power in case of over frequency. Data for scheduled power generation is taken from NRLDC site.

This data for the previous generation blocks frequency, along with data for scheduled power, actual power generated and declared capacity of that generating unit is utilized for training ANN to predict the frequency. Data from RLDC center/NRLDC and one of the power Generation unit is being taken for which frequency prediction will be made from the period Jan, 2015 to April 2017.Two years data i.e. from Jan, 2015 to Jan, 2017 will be used for training and 3 months data from Feb, 2017 to April, 2017 will be used for validation and testing Neural Network model [10], [11] for optimal performance.

Based on the ANN training the data in one block for all previous days will be taken and corresponding frequency for the same block will be predicted. Generation data for previous days scheduled generation; suggested generation and frequency of that block will be taken as input to ANN for training /learning of ANN.

ANN model performance is realized with set of parameters such as learning rate, momentum rate, number of layer and number of neuron and most appropriate model is chosen for representing the network, which will optimal frequency prediction. we will keep on validating the above parameters ,till we get a sustained output for a chosen set of the ANN model.

\section{ARTIFICIAL NEURAL NETWORK BASED APPROACH FOR FREQUENCY FORCASTING}

Frequency forecasting is a pattern matching and generalization problem. ANN performs reasonably well for such problems. ANN model depends on mapping of input output spaces and therefore are free from explicit defined frequency models from which the statistical methods suffer from.ANN has versatile capability for adaptation and performs most appropriately for such realizations of Random Frequency clusters. ANN overcomes the limitation of the linear network and is able to model non-linear relationship of the multilayer feed forward network with learning carried out using back propagation rule. Typically, neurons are organized in layers. Different layers may perform different kinds of transformations on their inputs. Signals travel from the first (input), to the last (output) layer, possibly after traversing the layers multiple times. In ANN with multiple hidden layers, the initial layers might detect primitives and their output is fed forward to deeper layers who perform more abstract generalizations and so on until the final layers perform the complex object recognition. A typical ANN architecture shown in Fig..2.

The original goal of the neural network approach was to solve problems in the same way that a human brain would. Over the time, attention focused on matching specific mental abilities, leading to deviations from biology such as back propagation, or passing information in the reverse direction and adjusting the network to reflect that information. Normally a neural network is trained to forecast the frequency up to predefined lead-time. It can be a lead of one day or a week ahead.

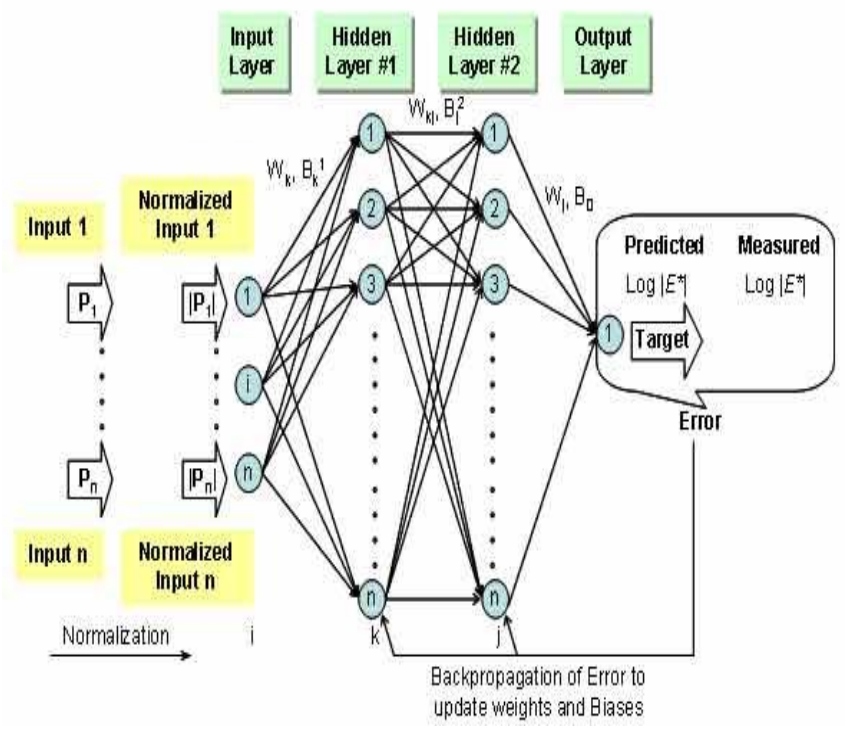

Fig.2: Artificial Neural Network Architecture 


\subsection{Multilayer Feed Forward Network with Back}

\section{Propagation Learning Algorithms}

Multi-layer networks uses a variety of learning techniques, the most popular being back-propagation. Here, the output values are compared with the correct answer to compute the value of some predefined error-function. By various techniques, the error is then fed back through the network. Using this information, the algorithm adjusts the weights of each connection in order to reduce the value of the error function by some small amount. After repeating this process for a sufficiently large number of training cycles, the network will usually converge to some state where the error of the calculations is small. In this case, one would say that the network has learned a certain target function. To adjust weights properly, one applies a general method for nonlinear optimization that is called gradient descent. For this, the network calculates the derivative of the error function with respect to the network weights, and changes the weights such that the error decreases (thus going downhill on the surface of the error function). For this reason, back- propagation can only be applied on networks with differentiable activation functions.

In general, the problem of teaching a network to perform well, even on samples that were not used as training samples, is a quite subtle issue that requires additional techniques. This is especially important for cases where only very limited numbers of training samples are available 12$]$. The danger is that the network over fits the training data and fails to capture the true statistical process generating the data. Computational learning theory is concerned with training classifiers on a limited amount of data. In the context of neural networks a simple heuristic, called early stopping, often ensures that the network will generalize well to examples not in the training set. Other typical problems of the back-propagation algorithm are the speed of convergence and the possibility of ending up in a local minimum of the error function. Today there are practical methods that make back-propagation in multi-layer perceptions the tool of choice for many machine learning tasks.

\section{Backpropagation Algorithm}

\section{Before Weight Adjustment}

$$
\begin{aligned}
& \text { Parameters } \\
& \text { For } w_{2}=5 \wedge x=2 \wedge w_{1}=3.5 \\
& \text { Where } M A E_{1}=w_{1} x-y \wedge M A E_{2}=w_{2} x-y \\
& \text { For } w_{2} x=10 \wedge w_{1} x=7 \wedge y=4 \\
& f(x)=\frac{M A E_{2}^{2}}{2} \\
& g(x)=\frac{M A E_{1}^{2}}{2} \\
& f(x)=\frac{E_{2}^{2}}{2} \\
& \text { Chain Rule } \\
& \frac{\partial g}{\partial x}=\frac{\partial g}{\partial f} \frac{\partial f}{\partial x}=g^{\prime}(x)=g^{\prime}(f(x)) \cdot f^{\prime}(x) \\
& \frac{\partial f}{\partial x}=f^{\prime}(x)=2 \cdot \frac{E_{2}}{2}=E_{2}=w_{2} x=10 \\
& \frac{\partial g}{\partial x}=\frac{\partial g}{\partial f} \frac{\partial f}{\partial x}=g^{\prime}(x)=g^{\prime}\left(f\left(w_{1} x-y\right)\right) \cdot f^{\prime}(x) \\
& g^{\prime}(x)=g^{\prime}(f(3)) \cdot 10 \\
& g^{\prime}(x)=\frac{1}{2} \cdot 3^{2} \cdot 10=\frac{90}{2} \text { 45 } \begin{array}{l}
\text { Derivative } \\
\text { of error }
\end{array} \\
& g(x)=\frac{E_{1}^{2}}{2} \\
& \frac{\partial g}{\partial x}=\frac{\partial g}{\partial f} \frac{\partial f}{\partial x}=g^{\prime}(x)=g^{\prime}(f(x)) \cdot f^{\prime}(x) \\
& g^{\prime}(x)=g^{\prime}\left(f\left(w_{1} x-y\right)\right) \cdot f^{\prime}(x) \\
& g^{\prime}(x)=g^{\prime}(f(1)) \cdot 8 \text { Derivative } \\
& \text { of error } \\
& g^{\prime}(x)=\frac{1}{2} \cdot 1^{2} \cdot 8=\frac{8}{2}=4 \begin{array}{l}
\text { After } \\
\text { Backprop }
\end{array}
\end{aligned}
$$

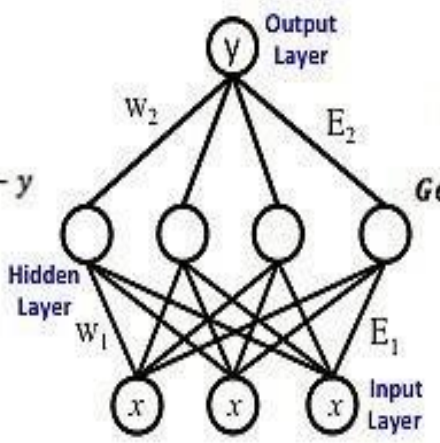

After Weight Adjustment

Fig.3: MLBPN 


\subsection{Radial Basis Function Network}

A Radial Basis Function (RBF) Network consists of two layers, a hidden layer with nonlinear neurons and an output layer with linear neurons. Thus the transformation from the input space to the hidden unit space is non-linear whereas the transformation from the hidden unit space to the output space is linear. The basis functions in the hidden layer produce a localized response to the input i.e. each hidden unit has a localized receptive field. The basis function can be viewed as the activation function in the hidden layer.
This is becoming an increasingly popular neural network with diverse applications and is probably the main rival to the multi-layered perception. RBF trains faster than a MLP.Another advantage that is claimed is that the hidden layer is easier to interpret than the hidden layer in an MLP. The most commonly used radial-basis function is a Gaussian function. It trains and simulates the faster than other MLBPN algorithms the basic model of a RBF network is shown in Fig.5

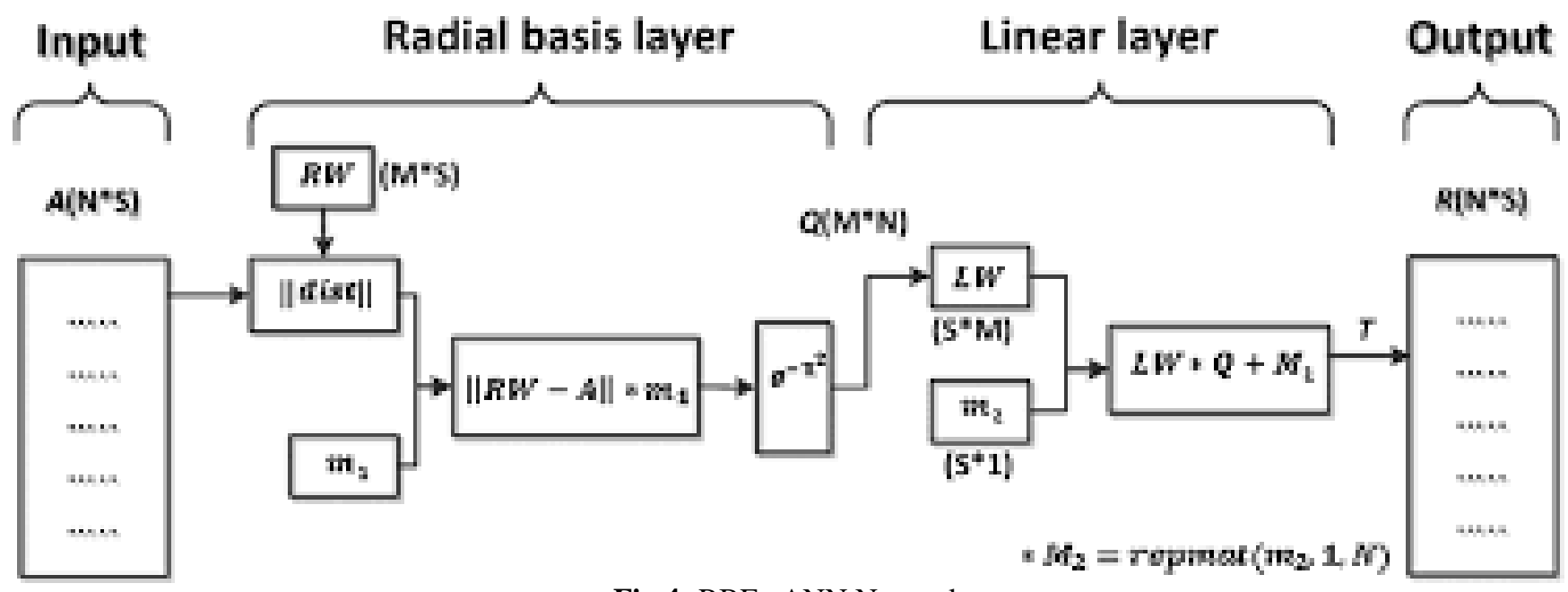

Fig.4: RBF -ANN Network

\subsection{Feature Selections and Network Architecture}

Feature and parameter selection is made in unified manner which includes recognition of the set of dependent and independent variables from learning data that can outline input and output spaces in optimal ways. Numerous input variables are possible which can represent input space for defined problem. Some of them may have strong analogy with other input and output spaces and rest may have least effect on it. This feature selection enable them to select one set of input /output space variable that have greater impact on its output for a defined task to accomplish. The sample data comprises of training data and validation/pattern testing data. This procedure starts with a single set variable space. When a network model is in shape after training, it is tested with the validation data before actual putting in any application. A typical flow chart for given set of input and output variable spaces is shown in Fig.5

Forecasting of the frequency for ahead time depends on prior frequency, weather condition along with the other parameter of the external influence too.

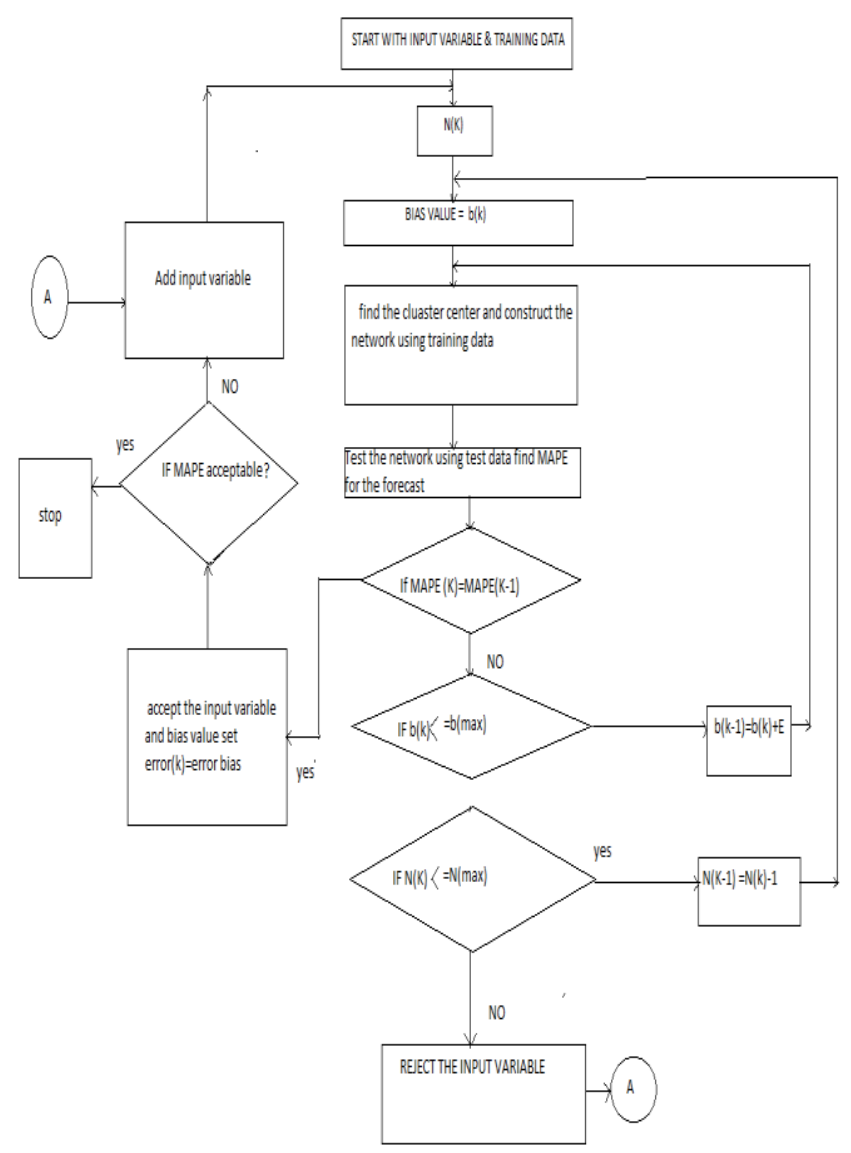

Fig 5: Flow Chart for Feature and architecture selection 


\subsection{ANN Training and Sample Data}

After every 15 minutes data is being recorded, which includes time, frequency, Power Generated in MW, Scheduled Generation in MW from RLDC site and Actual Power generation in MW.For the Frequency predication at new time instances ANN will be used based on past data analysis. We have taken the data for frequency, scheduled generation and available generation of 2 years and 3 months .For learning of the ANN we use the data of 96 block from Jan,2015 to Jan ,2017 (24 months ) and three months data is used for validation and testing of the network. Table.1 shows the sample data of the selected day (for 21 blocks).

Table 1: ANN Training data

\begin{tabular}{|l|l|l|l|l|}
\hline Time & Block & Freq. & Gen.(MW) & \multirow{2}{*}{ Sch.(MW) } \\
\hline & No. & $\mathbf{( H z . )}$ & & \\
\hline $0: 15$ & 1 & 50.06 & 304.130 & 335.38 \\
\hline $0: 30$ & 2 & 50.03 & 300.100 & 335.38 \\
\hline $0: 45$ & 3 & 50.05 & 291.020 & 335.38 \\
\hline $1: 00$ & 4 & 50.06 & 272.910 & 335.38 \\
\hline $1: 15$ & 5 & 50.02 & 258.200 & 349.35 \\
\hline $1: 30$ & 6 & 50.02 & 252.150 & 349.35 \\
\hline $1: 45$ & 7 & 50.01 & 251.050 & 349.35 \\
\hline $2: 00$ & 8 & 50.03 & 247.900 & 349.27 \\
\hline $2: 15$ & 9 & 50.02 & 247.480 & 328.18 \\
\hline $2: 30$ & 10 & 50.03 & 245.460 & 303.18 \\
\hline $2: 45$ & 11 & 50.00 & 248.990 & 298.10 \\
\hline $3: 00$ & 12 & 49.98 & 250.930 & 298.10 \\
\hline $3: 15$ & 13 & 49.92 & 269.350 & 349.01 \\
\hline $3: 30$ & 14 & 49.93 & 283.740 & 349.01 \\
\hline $3: 45$ & 15 & 49.94 & 279.560 & 349.01 \\
\hline $4: 00$ & 16 & 49.99 & 292.230 & 320.45 \\
\hline $4: 15$ & 17 & 50.00 & 276.140 & 290.45 \\
\hline $4: 30$ & 18 & 50.01 & 254.340 & 260.45 \\
\hline $4: 45$ & 19 & 50.00 & 239.850 & 245.00 \\
\hline $5: 00$ & 20 & 50.02 & 241.470 & 245.00 \\
\hline $5: 15$ & 21 & 50.03 & 239.460 & 245.00 \\
\hline & & & & \\
\cline { 1 - 2 } & & & \\
\hline
\end{tabular}

\subsection{Methodology}

Frequency forecasting is based on only historical frequency data of the system. The weather variables and their influence on system frequency were not considered due to unavailability of weather data. The ANN model could be used for predicting future weather conditions such as humidity, temperature etc. A combined model of ANN which first predicts the weather conditions and then forecasts the frequency could greatly improve the accuracy of frequency forecasts The input to the network comprised of historical block generation frequencies concerning to the first case for a week grouped into vectors of 12 elements each. In MLP structure a network comprising of 3 hidden layers and having learning and momentum rates $0.2 \& 0.6$ respectively has been chosen. The three hidden layers were having $16,14 \& 12$ neurons respectively. The sum-squared error goal for this network was 0.001 . In the second case a network with 3 hidden layers and having 14, 12\&10 neurons respectively and having a learning rate of $0.3 \&$ momentum rate of 0.5 has been chosen. In third case a network structure with 3 hidden layers, having 12, $10 \& 8$ neurons a learning rate of $0.4 \&$ momentum rate of 0.6 has been chosen. Once these networks were trained frequencies for next 24 hours/96 blocks of the desire day will be predicted. These results are shown in tabular form in Table $2 \& 3$ and graphically in Fig.8 \&9.

A python programming is used for the ANN model design as it significantly support the web based applications for picking up the generation schedule from RLDC site and actual power generation from server of the power generating unit.

\section{RESULTS}

\subsection{Result (Case-I)}

Simulation Result of Predicted Block Frequency with Multilayer Feed Forward Network without considering the generation schedule and declared capacity.

[50.049, 49.939, 49.909, 50.0, $50.029,49.979,50.06,49.959,256.36,49.97,49$.

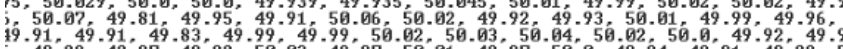

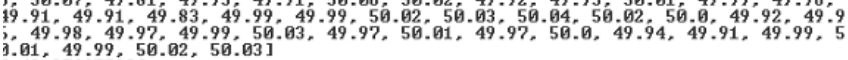
1. 49.979 .996533

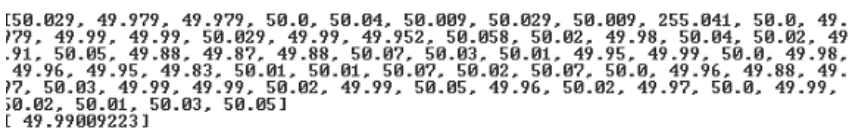

[50.8, 49.99, 50.849, 50.02, 50, $02,50.009,50.029,50,029,256.25,50.0,49.979$

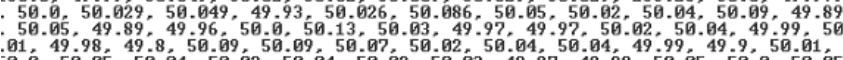

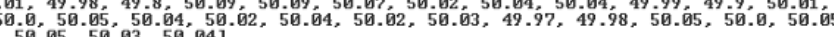
$50.05,50.93,50.041$
50.012752341

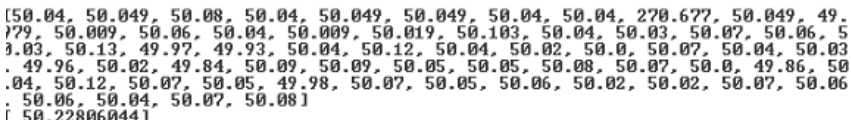

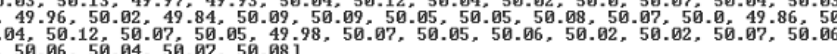
i $50.06,50.628644$.

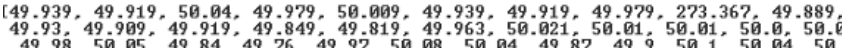

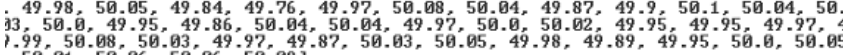

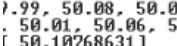

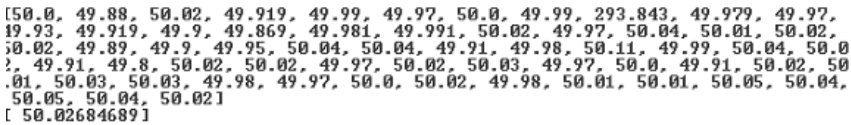

[50.009, $49.97,50.02,49.979,49.97,49.97,50.0,50.029,319.365,49,99,49.96$ $13,50.01,49.89,49.88,49.95,50.02,56.01,49.89,49.96,50.1,50.01,50.04$,

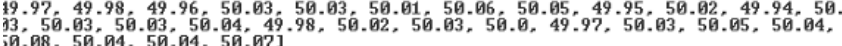

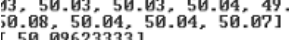




\subsubsection{Clockwise Numerical Values of the Previous}

\section{Days and Predicted Frequencies}

\section{Block-1}

INPUT:[49.95, 50.06, 49.979, 49.919, 50.04, 49.979, 49.99, 49.97, 49.99, 50.029, 49.95, 49.959, 49.99, 49.939, 49.975, $50.005,50.03,50.04,50.07,50.02,50.02,49.98,50.09$, $50.05,49.84,49.99,50.08,49.96,50.03,50.01,50.03$, $50.04,50.01,49.94,49.98,50.0,50.0,50.02,50.03,50.08$, $50.03,50.02,50.02,49.99,49.94,50.04,50.04,50.04$, 49.91, 49.98, 50.0, 49.99, 49.93, 50.0, 50.0, 49.93, 50.06, 50.03, 50.04],OUTPUT: [50.0040709]

\section{Block-2}

INPUT:[49.93，50.049，49.99，50.029，50.06，50.029, 50.009, 50.009, 50.009, 50.049, 49.99, 49.99, 50.029, 49.93, 49.996, 50.006, 50.04, 50.01, 50.06, 49.99, 50.05, 50.0, 50.1, 50.0, 49.9, 50.01, 50.1, 50.0, 49.96, 50.0, 50.05, 50.02, $50.02,49.98,50.01,50.01,50.01,50.0,50.03,50.04,50.01$, 50.04, 50.01, 49.97, 50.0, 50.03, 50.02, 50.04, 49.93, 50.01, $50.01,50.03,50.01,49.99,50.01,49.98,50.04,50.08$, 50.03],OUTPUT: [ 50.01297654]

\section{Block-3}

INPUT:[49.909, 50.04, 49.99, 50.02, 50.04, 50.0, 49.979, 50.02 , 50.009, 50.08, 50.02, 49.93, 50.0, 49.909, 49.959, $49.996,50.03,50.02,50.06,50.02,50.03,50.0,50.17$, 50.02, 49.89, 50.0, 50.09, 50.03, 49.98, 49.99, 50.06, 50.02, $50.04,49.98,49.99,49.98,49.98,50.01,50.03,50.05$, $50.02,50.03,50.01,49.98,49.93,50.03,50.03,50.02$, $49.99,49.98,49.98,50.01,49.99,50.02,50.02,49.99$, 50.05, 50.02, 50.04],OUTPUT: [ 50.01041206]

\section{Block-4}

NPUT:[49.97, 50.04, 50.029, 50.009, 50.02, 50.04, 50.009, $50.009,50.02,50.08,50.029,50.009,50.009,49.95,50.0$, $49.983,50.03,50.04,50.03,50.04,50.04,50.02,50.1$, $50.01,49.94,50.02,50.07,50.02,49.98,49.96,50.12$, $50.03,50.0,50.04,50.03,50.02,50.02,50.0,50.04,50.02$, $50.02,50.02,50.05,50.01,50.03,50.03,50.04,50.01$, $49.99,50.02,50.01,50.05,50.01,50.0,50.03,50.0,50.06$, $50.03,50.01]$

OUTPUT: [50.02222542]

\section{Block-5}

INPUT:[49.979, 50.029, 49.979，50.02，50.049，50.029, 50.009 , 50.02, 50.04, 50.08, 50.02, 50.02, 50.009, 49.99, $50.01,50.003,50.03,50.05,50.04,50.06,50.05,50.0$, $50.05,50.03,49.98,50.02,50.08,50.02,50.03,50.01$, $50.08,50.02,50.03,50.04,50.05,50.01,50.01,49.99,50.0$, $50.03,50.05,50.02,50.05,50.02,49.97,50.02,50.03$, $50.05,49.97,50.03,50.01,50.01,50.01,50.01,50.01$, 50.02, 50.03, 50.03, 50.02],OUTPUT: [ 50.02490854]

\section{Block-6}

INPUT:[50.02, 50.02, 49.95, 50.029, 50.029, 50.06, 50.04, 50.029, 50.029, 50.06, 50.02, 50.029, 50.02, 49.979, 49.997, $50.023,50.06,50.03,50.06,50.05,50.05,50.02,50.05$, $50.04,49.96,50.02,50.08,50.01,50.03,50.04,50.08$, $50.01,50.0,50.05,50.02,50.02,50.02,50.0,50.01,50.02$, $50.03,50.02,50.03,49.99,50.02,50.02,50.04,50.02$, 49.98, 50.03, 50.02, 50.01, 49.94, 50.0, 50.03, 50.03, 50.05, 50.04, 50.0]OUTPUT: [ 50.02277562]

\subsubsection{Graphical Representation}

Red line Input frequencies of a Block Blue line Predicted frequency of Block

\section{Block-1}

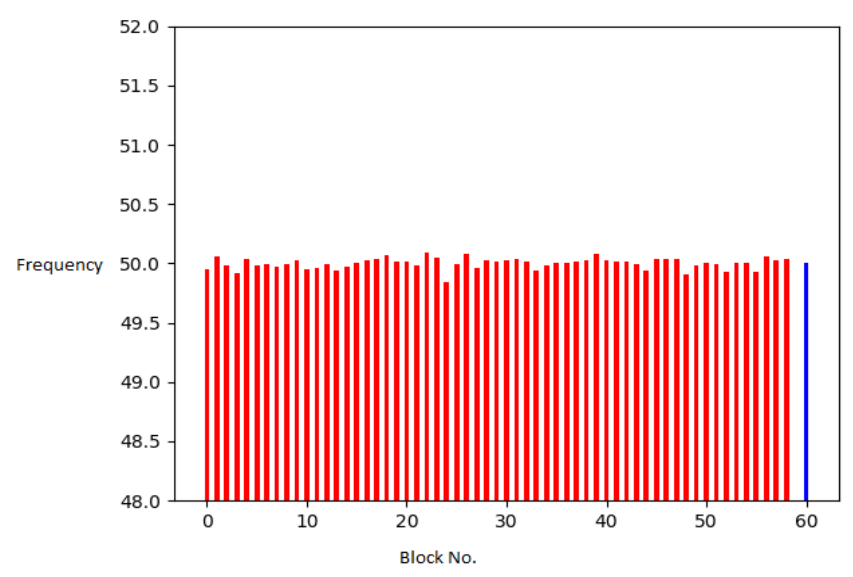

Fig.6: Predicted frequency(Block-1)

\section{Block-2}

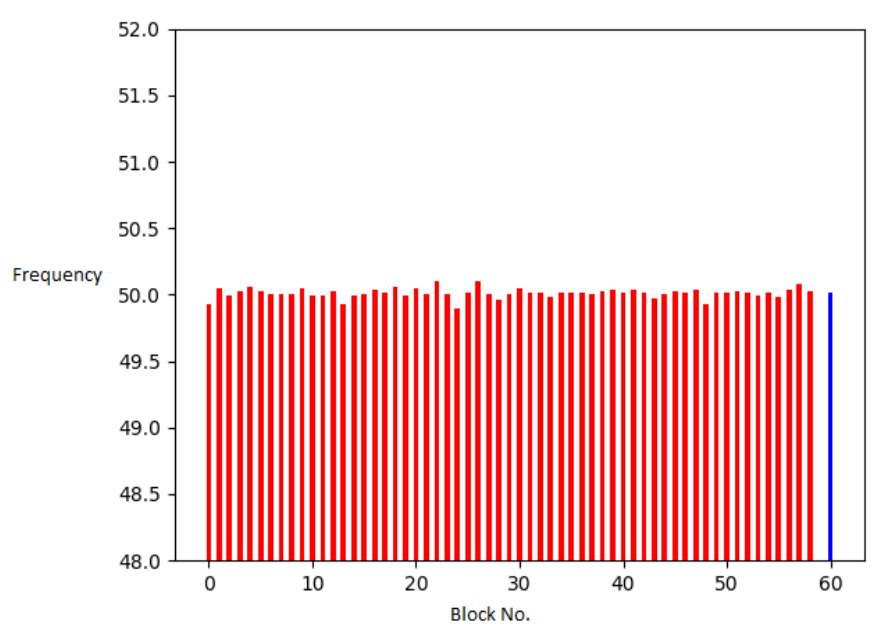

Fig.7: Predicted frequency(Block-2

\subsection{Result (Case-II): Frequency Prediction with MLBPN Network with Generation Data (Schedule} Generation \& Unit Capacity)

Table 2: Actual and Predicted

\begin{tabular}{|l|l|l|l|}
\hline Block No. & $\begin{array}{l}\text { Predicted } \\
\text { Frequenc } \\
\text { y }(\mathbf{H z})\end{array}$ & $\begin{array}{l}\text { Actual } \\
\text { freque } \\
\text { ncy }(\mathbf{H}\end{array}$ & $\begin{array}{l}\text { \% Absolute } \\
\text { Error }\end{array}$ \\
\hline
\end{tabular}




\begin{tabular}{|l|l|l|l|}
\hline & & z) & \\
\hline Block-1 & 50.072 & 50.049 & 1.99725 \\
\hline Block-2 & 50.027 & 50.009 & 1.79905 \\
\hline Block-3 & 49.968 & 50.009 & 4.1025 \\
\hline Block-4 & 50.06 & 50.049 & 1.0987 \\
\hline Block-5 & 50.01 & 50 & 0.9998 \\
\hline Block-6 & 50.07 & 50.029 & 4.09425 \\
\hline Block-7 & 50.1 & 50.009 & 9.08185 \\
\hline Block-8 & 50.11 & 50.009 & 10.07785 \\
\hline Block-9 & 50.25 & 50.099 & 15.0249 \\
\hline Block-10 & 50.08 & 49.939 & 14.0775 \\
\hline Block-11 & 49.98 & 50.04 & 6.0025 \\
\hline Block-12 & 50.06 & 49.95 & 10.9868 \\
\hline Block-13 & 50.12 & 50.049 & 7.083 \\
\hline Block-14 & 50.25 & 50.109 & 14.02985 \\
\hline Block-15 & 50.07 & 50.029 & 4.09425 \\
\hline Block-16 & 50.1 & 50.009 & 9.08185 \\
\hline Block-17 & 50.11 & 50.009 & 10.07785 \\
\hline Block-18 & 50.25 & 50.219 & 3.0846 \\
\hline Block-19 & 50.12 & 50.049 & 7.083 \\
\hline Block-20 & 50.25 & 50.109 & 14.02985 \\
\hline Block-21 & 50.07 & 50.029 & 4.09425 \\
\hline Block-22 & 50.069 & 50.049 & 1.99725 \\
\hline Block-23 & 50.027 & 50.009 & 1.79905 \\
\hline Block-24 & 49.968 & 50.009 & 4.1025 \\
\hline
\end{tabular}

\section{Frequency Case-II}

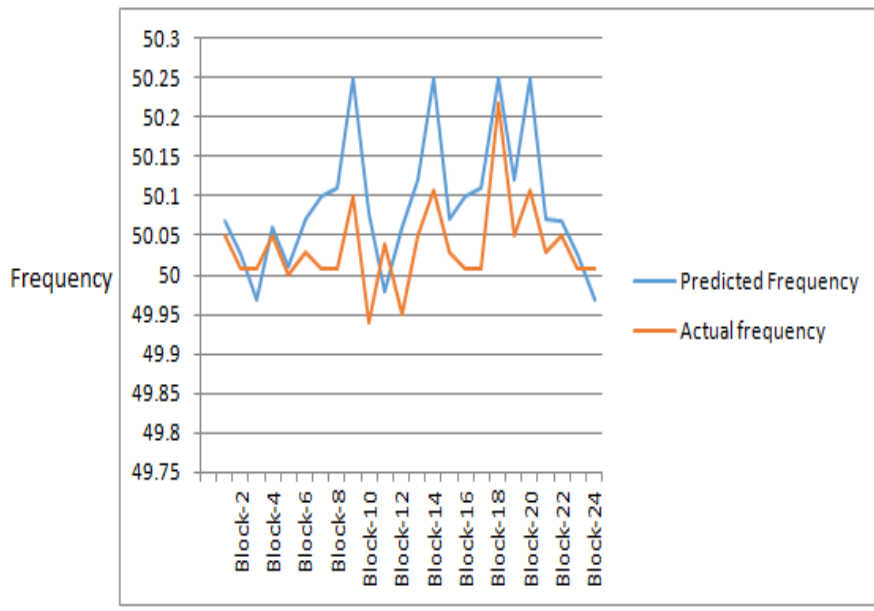

Fig 8: Graph between actual and predicted frequency-CASE II

\subsection{Result (Case-III): Frequency Prediction with}

\section{Radial Basis Function Network}

Table 3: Actual and predicted frequency

\begin{tabular}{|l|l|l|l|}
\hline Block No & $\begin{array}{l}\text { Predicted } \\
\text { Frequency }\end{array}$ & $\begin{array}{l}\text { Actual } \\
\text { Frequency }\end{array}$ & $\begin{array}{l}\text { \% Absolute } \\
\text { Error }\end{array}$ \\
\hline Block-1 & 50.069 & 50.049 & 0.964704 \\
\hline Block-2 & 50.027 & 50.009 & 4.944595 \\
\hline Block-3 & 49.968 & 50.009 & 4.901697 \\
\hline Block-4 & 50.06 & 50.049 & 0.906309 \\
\hline
\end{tabular}

\begin{tabular}{|l|l|l|l|}
\hline Block-5 & 50.01 & 50 & 5.834069 \\
\hline Block-6 & 50.07 & 50.029 & 3.007696 \\
\hline Block-7 & 50.1 & 50.009 & 5.102865 \\
\hline Block-8 & 50.11 & 50.009 & 5.219078 \\
\hline Block-9 & 50.25 & 50.129 & 3.690668 \\
\hline Block-10 & 50.08 & 49.999 & 12.39749 \\
\hline Block-11 & 49.98 & 50.04 & 2.406848 \\
\hline Block-12 & 50.06 & 49.95 & 11.56018 \\
\hline Block-13 & 50.12 & 50.049 & 1.786684 \\
\hline Block-14 & 50.25 & 50.109 & 4.206029 \\
\hline Block-15 & 50.07 & 50.029 & 3.73132 \\
\hline Block-16 & 50.1 & 50.009 & 5.577104 \\
\hline Block-17 & 50.11 & 50.009 & 5.496241 \\
\hline Block-18 & 50.25 & 50.129 & 15.62925 \\
\hline Block-19 & 50.08 & 49.939 & 1.248171 \\
\hline Block-20 & 50.04 & 50.08 & 4.825042 \\
\hline Block-21 & 49.95 & 49.98 & 3.196471 \\
\hline Block-22 & 50.049 & 50.06 & 0.964704 \\
\hline Block-23 & 50.109 & 50.12 & 4.944595 \\
\hline Block-24 & 50.029 & 50.25 & 4.901697 \\
\hline
\end{tabular}

\section{Case III}

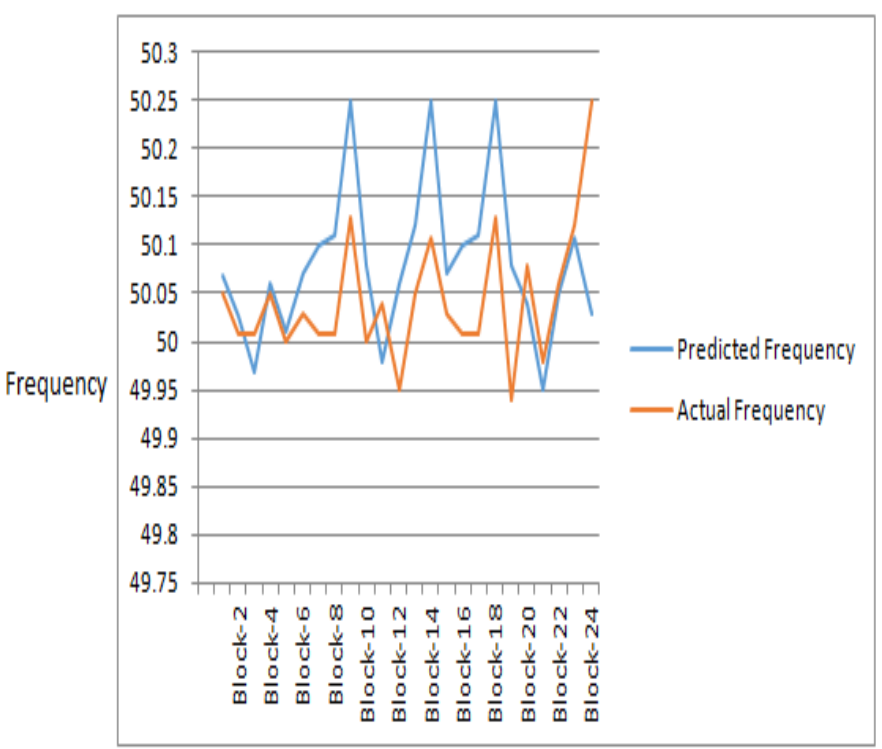

Fig 9: Forecasted Results with RBF Network Case III

\section{CONCLUSION}

[1] ANN model gives an important tool for short term frequency prediction which is quite different from conventional statistical approaches. They have shown very encouraging results for block generation frequency prediction to a fair degree of accuracy.Once a model is identified for a given task the learning of the ANN algorithms is to be selected for better speed and reliability of the frequency forecasting. This paper study provides block wise (in span of every 15 minutes) frequency prediction which 
is fast and largely depends on the algorithm choice between the MLBPN and Radial basis function which needs to be selected prudently. MLBPN approach is best for its precision whereas RBF train and simulate faster. Results shown here in this study RBF Model approach is most appropriate for prediction span of every 15 minutes. All the ANN models are vulnerable to spurious data. For maintaining acceptable accuracy in frequency predicting, effective data filtering must be framed and associated with ANN model. On account of the lack of the availability of the accurate weather information, their effect on frequency prediction could not be considered.

[2] Based on the future time block generation frequency data, we can link the same with the real time power generating utility server where we can integrate actual power, frequency and suggested generation data on line. The opportunity utilization for maximizing earnings can be done in accordance with the predicted frequency and we can be prepared in advance for resource mobilization of meeting the opportunity utilization by over /under injection of the power in the grid.

\section{ACKNOWLEDGEMENT}

Author will like to thanks the department of the Electrical Engineering, KNIT, and Sultanpur for all their support and help.

\section{REFERENCES}

[1] A.K.Gupta, R.Balasubramanian and N Vaitheeswaran, "ANN-based block frequency prediction in $\mathrm{ABT}$ regime and optimal availability declaration," IEEE Power and Energy Society General Meeting-Conversion and Delivery of Electrical Energy in the 21st Century, pp. 1-8, 2008.

[2] S.Kaur,S.Agrawal and Y.P.Verma, "Power Grid Frequency Prediction Using ANN Considering the Stochasticity of Wind Power," 2013 5th International Conference and Computational Intelligence and Communication Networks, Mathura, 2013, pp. 311315.

[3] S R Deshmukh, D J Doke and Y P Nerkar, "Optimal generation scheduling under ABT using forecasted load and frequency," Power System Technology and IEEE Power India Conference, POWERCON 2008. Joint International Conference on, pp. 1-6

[4] G.Gross and F.D. Galiana, "Short Term Frequency Forecasting" Proc. IEEE, vol.75, no 12, Dec., 1987, pp.1558-1578

[5] S.Rahman and I Moghram, "Analysis and evaluation of five short term frequency forecast Techniques", IEEE Trans. On Power Systems, vol.4, no.4, 1989, pp. 1484-1491Hu Jia-bing, Sun Dan, He Yi-kang, “A Model and Control Method of the DFIG during Grid Voltage Dip". Automation of Electrical Power System, 2006, 30(8):21-26.
[6] M.T. Hagen and S.M.Behr, "The time series approach to time series frequency forecasting ", IEEE Trans, on Power Systems, PRWS-2(3), 1987, pp. 785-791.

[7] B. Bhushan B, "ABC of $\mathrm{ABT}_{-} \mathrm{A}$ primer on availability tariff," 2005 available on http://www.nrldc.org/nrldc/docs.asp

[8] "Electricity Act, 2003", available on http://www.nrldc.org/docs

[9] Indian Electricity Grid Code (IEGC), effective from April'2006, available on http://www.nrldc.org/docs/gridcode.pdfwww.nrldc.ac in

[10] http://www.nrldc.org/docs/gridcode.pdf www.nrldc.ac.in

[11] www.ntpc.co.in intranet site.

[12] Roman M. Balabin; Ravilya Z. Safieva; Ekaterina I. Lomakina (2007). "Comparison of linear and nonlinear calibration models based on near infrared (NIR) spectroscopy data for gasoline properties prediction". Chemometr Intell Lab. 88 (2): 183-188

\section{BIOGRAPHY}

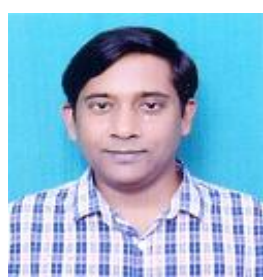

Shailendra Singh, B.Tech. (Electrical Engg.) \& MBA (Operation Management). He has 11 Years experience of Erection, commissioning and O\&M of various Electrical installations. His area of interest is Power System Operation and control. 